\title{
BREVES NOTAS SOBRE LA LENGUA TZOTZIL: LITERATURA ORAL Y CLASIFICADORES NUMERALES
}

por Antonio GARCía de LEÓN, Centro de Estudios Mayas, UNAM

El tzotzil, una de las lenguas mayances de los Altos de Chiapas, cuenta actualmente con cerca de 70000 hablantes. Se trata en realidad de una red de dialectos cuyas unidades principales de diferenciación coinciden con las cabeceras de comunidad más importantes de esta zona como son Chamula, Zinacantán, Chalchihuitán, Soyaló, Huistán y otras, las que a su vez dependen del comercio con el centro rector ladino de San Cristóbal Las Casas. El vecino y pariente lingüístico más próximo del tzotzil es el tzeltal, cuya red dialectal se toca en algunos puntos con la del primero.

En este trabajo presentamos dos de las características más interesantes de los materiales que hemos recopilado durante una temporada de campo en diciembre de 1970. La primera es una breve colección de algunos trozos narrados en lengua indígena; la otra es un inventario de los clasificadores numerales en tzotzil colonial y moderno, siendo éste uno de los aspectos gramaticales que comparte con la mayoría de las lenguas de esta gran familia.

En cuanto a la literatura oral, ésta funciona actualmente en muchas comunidades indígenas $\mathrm{y}$ aldeanas de Mesoamérica totalmente integrada a los sistemas de cohesión social, como suele ser la lengua indígena, los lazos de parentesco, las ligas religiosas, etcétera. Estos sistemas, cada vez más perturbados por la penetración de relaciones capitalistas provenientes de la ciudad, se desintegran día con día. Está de más decir que esta literatura oral muestra un gran arraigo de siglos y es obvio que en el medio en que florece tiene una función mucho más útil y extendida que la literatura escrita de las sociedades de consumo.

La muestra que aquí presentamos en su traducción al español tiene el valor intrínseco de ser trozos narrados por jóvenes alfabeti- 
zados que ya han tenido mucho más contacto con la vida de la ciudad que sus mayores. En este caso se trata de trozos mitológicos, legendarios y semihistóricos que fueron tomados al azar para poder efectuar un análisis sintáctico de esta lengua. No hay que olvidar que muchos de los famosos documentos coloniales del área maya (como el Popol Vij o los Chilames) son en realidad fragmentos de literatura oral indígena ya mezclada con algunas creencias de los conquistadores; y la motivación de los que las preservaron para nosotros fue también debida a un proceso de autodefensa de un mundo atrasado económicamente que aún pervive en las zonas rurales de nuestro país.

En cuanto a los clasificadores numerales, morfemas clasificadores que van unidos a la raíz de número, es claro que encierran en sí toda una visión del mundo; la clasificación por características del ambiente, objetos, personas y animales que rodean al hombre. También siguen reflejando en parte la preocupación que tuvieron los antiguos mayas por la medición del espacio y el tiempo.

Como apéndice final incluimos un cuadro con los meses del calendario agrícola de los Altos de Chiapas, con datos coloniales y modernos, que creemos puede ser ampliado por los especialistas en este campo.

1. Relatos de Chalchibuitán y Zinacantán. Los siguientes relatos proceden del paraje Sisim de San Pablo Chalchihuitán y fueron narrados por el joven Mario Gómez Girón; así como del paraje Paste' perteneciente a Zinacantán y narrados por el joven Juan Martínez.

\subsection{Chalcbibuitán}

"El Sol y la Luna"

Antiguamente los viejos oyeron de sus antepasados que Dios tuvo dificultades en su milpa cuando vivía en la tierra. Llegaba cada mañana a su milpa y veía que su trabajo no progresaba pues los pájaros lo destruían y se comían la semilla. Así Dios fue perseguido en la tierra y se escondía en las matas de plátano y en la tierra. Tanto lo hostilizaron que decidió hacer una escalera para subir al cielo; tardó diez días en llegar al cielo para iluminarlo. Tomax ("Tomás", el planeta Mercurio), su hermano, se hizo poner petróleo en la cabeza y subió encendido; Luis (lucero de la mañana) siguió también el ejemplo de sus hermanos porque temía que lo mataran. 
La madre ch'ulme'tik (la luna) decidió ir con sus hijos para no quedar sola en la tierra; pasaba por cada milpa orinando y donde orinaba crecía el chayote. Pasaba por otras milpas, enterraba el dedo y crecía el chilacayote. Construyó otra escalera y subió al cielo. Desde antiguo se pensó que era mejor que alumbrara la noche, y así se fue y resultó mejor. Otros dioses quedaron en la tierra y le ordenaron subir. La luna se fue llorando porque se fue sola al cielo.

\section{"El Diluvio"}

Los primeros hombres se empezaron a morir y algunos se refugiaron en los cerros. Se murieron casi todos de calentura, y los que lograron esconderse se volvieron monos. Llovió cuatro días seguidos anegándose toda la tierra y hasta los diez días descendió el agua. Quemaron todo y se quedó sola la tierra.

A los veinticinco días vinieron otros hombres del cielo y Dios les dijo: "No hay nada de comer." Entonces los hombres comían sólo cenizas y de ellas se mantenían. Dios bajó y dio un pedacito de tortilla a cada uno y con esto aguantaron un año. La luna se bajó a dejar un solo grano de maíz; de esto sembraron y volvieron nuevamente a surgir todos los bastimentos.

\section{"Cuento del Dueño de los Animales"}

El Angelito es el dueño de los animales y a él acudió el jabalí para pedirle permiso: "Quiero tu permiso para ir a la milpa a comer, dijo el jabalí"; y el permiso se le dio y salió a los campos. El jabalí no llegó porque lo mataron en el camino. Un largo trecho lo llevó el cazador cargando y el Ángel le mató a su perro. El Ángel iba como culebra y el cazador la mató.

Cuando el hombre llegaba a la puerta de su casa, vino el Ángel y lo mató. La mujer fue a levantar a su marido, y al verlo muerto se quedó llorando. Después de una hora, el cuerpo del cazador se hinchó y se reventó.

\section{"Cuento del Ach'eljol"}

Los protectores de la tierra cuidan a la gente en los caminos y viven bajo la superficie; el macho es ach'eljol y la hembra uch'um- 
jol. ${ }^{1}$ Un día, al estar cuidando a las personas que caían en los caminos, decidieron trabajar para que hubiera más niños e hicieron cuatro de ellos en barro: Domingo, Salvador, Sabastían y Pedro. Éstos salieron a cazar; Sebastián dijo haber visto un guajolote y junto con Salvador lo fueron a matar con escopeta y tirador. Lo mataron y empezaron a comerlo y pasados cuatro días todavía no terminaban. Llegaron Pedro y Domingo y comenzaron a pelear por el pavo. El acb'eljol es travieso y sus hijos se comían sólo a una paloma.

\section{"El Hombre con Cola"}

Dicen que había un hombre que tenía su cola, buscaba la fruta en el monte y la dejaba en cada casa; como a la gente no le gustaba, ahí mismo se pudría la fruta.

La gente de entonces le quitó la cola a aquel hombre. Se dice que un tal Pedro Álvarez guardaba la cola y la perdió hace como veinticinco años. Dicen que la cola se convirtió en venado y se fue a los montes.

"El Hombre que se volvió zopilote"

Un hombre muy flojo quería ser zopilote para no trabajar. Dijo: "Mejor quiero ser zopilote." Dio cuatro brincos en el fogón; se cayó herido con un ojo cerrado y a las cinco veces se fue volando a buscar que comer. Su papá decidió hacer lo mismo, se fue a rezar a la cueva y pidió ser tigre; salió convertido en tigre y se fue contento al monte.

\subsection{Zinacantán}

"La Iglesia de Zinacantán"

Antiguamente, cuando no había iglesia, se oscureció tres días y tres noches. Cuando amaneció, Dios paró la iglesia y las casas de San Lorenzo y San Sebastián; la casa del último se cayó y la de San Lorenzo no.

En ese entonces no andaba Dios por la tierra, sólo San Lorenzo. Después apareció el Cristo en un lugar llamado Loksantó' cerca de

1 ach'-el jol es "cabeza lodosa"; uch'-um jol algo así como "cabeza de pozol". 
Pasté; apareció dentro del tronco de un árbol, en la orilla de una cueva. Partieron el tronco y se llevaron al Cristo a la iglesia de Zinacantán; le crecía la barba y la vendían a cien pesos. Los viejitos notaron que esto no estaba bien y lo mandaron a arreglar. Después ya no era el mismo, notaron que había cambiado porque ya no le crecía la barba; se percataron de que ya no era el mismo.

Después de eso, fueron a sacar del cerro una campana para la iglesia de San Lorenzo. No podían sacarla sino hasta después de mucho esfuerzo, la sacaron casi a las doce del día. La dejaron a la orilla de la cueva y se pusieron a comer. Unas señoras que busca ban leña se orinaron a la orilla de la cueva; se sumió más la campana y ya no la pudieron sacar.

\section{"El Eclipse de Luna"}

Las personas que no saben y miran la luna durante el eclipse, piensan que la va a matar la estrella muk'ta k'anal (Venus) porque se pelean.

Cuando miran esto empiezan a gritar, truenan escopetas, sacan sus flautas y tambores para que se espante el diablo, la estrella; y los viejitos sacan palos, se pegan a sí mismos y toman aguardiente para no sentir el momento de su muerte. También piensan que con esto ayudan a la luna para que no le siga pegando el diablo, y también para ahuyentar a éste.

"El que carga la tierra"

Dicen que la tierra es como una pelota y está sostenida por una persona (Vakbanej). Dicen que como la tierra está así sostenida, cuando la persona mueve la cabeza o alguna parte del cuerpo, la tierra empieza a moverse y sobreviene el temblor de tierra.

\section{"El Maíz}

Dicen que al maíz lo encontraron en una cueva y que estaba en poder de un mono; a éste lo mataron y le quitaron las mazorcas que tenía.

Sucedió que lo vieron los cazadores en la puerta de la cueva y 
lo siguieron hasta el interior, donde lo mataron. Fue así que trajeron las mazorcas a los pueblos.

\section{Los clasificadores numerales del tzotzil colonial y moderno ${ }^{2}$}

2.0. El sistema numeral del tzotzil es vigesimal. Las raíces numerales (pertenecientes a la clase de las Partículas) no son independientes, sino que forman compuestos radicales al unirse con los clasificadores numerales. Estos en su mayoría provienen de raíces verbales y nominales.

Otros numerales son reduplicados, como: ju-jun "cada"; o con sufijos como -ej, -je "tiempo en días" (pasado, futuro): cha-b-je "hace dos días", cha'-ej "dentro de dos días"; o el sufijo $-u k /-u$ "circunstancia específica": ch-ib-uk "sólo dos", j-set-uk "sólo unos cuantos".

Las raíces numerales, tal como preceden a los clasificadores son las siguientes:

$\begin{array}{ll}\text { 1. j- } & \text { 11. buluch- } \\ \text { 2. cha'- } & \text { 12. lajcha- } \\ \text { 3. ox- } & \text { 13. oxlajun- } \\ \text { 4. chan- } & \text { 14. chanlajun- } \\ \text { 5. jo' } & \text { 15. jo'lajun- } \\ \text { 6. vak- } & \text { 16. vaklajun- } \\ \text { 7. juk- } & \text { 17. juklajun- } \\ \text { 8. vaxak- } & \text { 18. vaxaklajun- } \\ \text { 9. balun- } & \text { 19. balunlajun- } \\ \text { 10. lajun- } & \text { 20. tob-ta- }{ }^{3}\end{array}$

2.1. Los clasificadores numerales en sí se pueden dividir en dos subclases (determinadas por el fonema inicial y por la raíz numeral a la que acompañan).

2.1.1. A la primera subclase pertenece el clasificador $-V b$ "clasificador numeral indeterminado" (que no aparece en el uno, el veinte y el cuatrocientos) y que define a los numerales ordinales indeterminados. Ejemplo:

${ }^{2}$ Los fonemas del tzotzil son: $\mathrm{p}, \mathrm{t}, \mathrm{ts}, \mathrm{ch}, \mathrm{k}, \mathrm{p}^{\prime}, \mathrm{t}^{\prime}, \mathrm{ts}^{\prime}, \mathrm{ch}^{\prime}, \mathrm{k}^{\prime},{ }^{\prime}, \mathrm{b}, \mathrm{s}, \mathrm{x}, \mathrm{j}, \mathrm{m}, \mathrm{n}$, $\mathrm{v}, \mathrm{y}, 1, \mathrm{r} ; \mathrm{i}, \mathrm{e}, \mathrm{a}, \mathrm{o}, \mathrm{u}$.

3 En esta ocasión no marcamos las divisiones morfémicas de las raíces numerales, pero son bastante obvias. 

1. jun
11. buluch-ib
2. $c h-i b$
12. lajch-eb, lajcha-y-eb
3. $o x-i b$
13. oxlajuneb
4. chan-ib
14. chanlajun-eb
5. $j o^{\prime}-o b$
15. jo'lajun-eb
6. vak-ib
16. vaklajun-eb
7. $j u k-u b$
17. juklajun-eb
8. vaxak-ib
18. vaxaklajun-eb
9. balun-eb
19. balunlajun-eb
10. lajun-eb
20. $t o b$

2.1.2. A la segunda subclase pertenecen 35 clasificadores derivados de raíces verbales y nominales, y que empiezan con consonante (a excepción del primero). Son los siguientes:

\section{CLASIFICADORES}

1. $-a b$ "de años"

2. -bus "de granos pilados"

3. (XVIII) -chop "de pares"

4. -ch'ix "de varas" (del pulgar al cordial)

5. $-c b^{\prime} \circ j$ "de agujeros"

6. -ch'ut "de jemes"

7. (XVIII) -y-ep-al, -ep-al "de cosas"

8. -jek "de piernas o cuartos de carne"

9. -jov "de brazadas o mitades"

10. -kep "de pencas de plátano"

11. -koj "de pisos"

12. -kot "de animales u objetos de cuatro patas"

13. -k'ol "de cuartos o generaciones"

14. (XVIII) -lik-el "de veces"

15. -lok' "de hilos"

16. -lom "de tandas de música o capas"

\section{ORIGEN VERBAL NOMINAL}

jab-il "año"

bus-an "amontonar grano o fruta"

-chap-al "clase", "familia"

ch'ix "espina"

$c b^{\prime} \circ j-$ "perforar"

ch'ut-ub "jeme"

$y$-ep-al "cantidad"; $e p$ - aj "aumen-

tar"

-jek-el "pierna"

jav-el "rajarse", "cortar por

mitad"

kep-el "penca"

kaj-al "encima de", kaj tsaj

"cubierto"

kot-ol "en cuatro patas", kot-om

k'al-el "rajar", "separar"

"tejón"

lik-el "principiar, tener, llevar" lok'-ol "hilo"

lom-el "caerse", lom-ol "vacío" 
17. (Chm) -mek "de viajes"

18. -moj "de veces o vueltas"

19. -pets "de plantas o pisos"

20. - poj "de racimos"

21. -p'ej, (Chm) -bej "de cosas redondas"

22. -p'el "de palabras"

23. - $p$ 'is "de medidas"

24. - $p$ 'os "de rayas"

25. - sets" "de medidas" (cazuelitas)

26. -tel "de atados"

27. (Chm) -ten "de cosas planas y delgadas"

(Cha) "de veces"

28. -tek" "de árboles, plantas, postes"

29. (XVIII) -tuk "de cantidades

30. - um "de bocados" pequeñas"

31. -vit "de plantas"

32. -vo' "de personas"

33. -xot "de cosas circulares"

34. -xut "de mitades"

moj-ol, moj-uk "otra vez" pets- "sentarse en el suelo" poj-ol "hueco", "racimo" p’ej-el "cosa redonda"

p'el-el "palabra, grito"

$p$ 'is "medir", "copa"

p'os-el "raya", p'as-as-t-ik "rayado"

-set "rebanar circularmente"

tel-om "andar derecho", tel-tel "rollizo"

ten-ub-il "cubrir, aplastar"

te' "árbol, palo, madera"

(XVIII) jun-tuk "un poco"

um-el "sorbo, bocado"

j-vit "cierta planta"

$v a^{\prime}-i$ "pararse", vinik "persona"

xot- "formar círculo"

xut- "partir por mitad"

35. -yom "de rollos o manojos"

yom-el "enrollar", yom-ol "junto"

La mayoría de los datos coloniales arriba anotados están tomados del "Anónimo" de 1832, que trata en parte de los antiguos materiales del Padre Manuel Hidalgo (1735); así como de la obra de De Rodaz (1688).

La abreviatura $(\mathrm{Chm})$ significa que se trata de un término sólo usado en Chamula; y (Cha), que se usa en Chalchihuitán.

2.2. Después de veinte, la palabra tob es sustituida por vinik "persona" para significar "veintena"; apareciendo éste en el sitio sintáctico de un clasificador: cha'-vinik "cuarenta", ox-vinik "sesenta", chan-vinik "ochenta". Algunos ejemplos de cuenta arriba de veinte son: (para mejor información se puede ver Hopkins, 1967).

22. ch-ib-x-cha'-vinik "contando dos hacia cuarenta"

39. balun-lajum-eb-x-cha'-vinik "contando diecinueve hacia cuarenta"

44. chan-y-ox-vinik "contando cuatro hacia sesenta" 
361. jun-s-balum-lajun-vinik "contando uno hacia diecinueve veintes $(380) "$

400. j-bok' "un zonte"

401. $j$-bok'-x-chik-jun "cuatrocientos con uno"

500. $j$-bok'-x-chik-jo'-vinik "cuatrocientos con cinco veintenas"

800. cha'-bok' "dos zontes"

804. cha'-bok-x-cbik-chan "dos zontes con cuatro" 1000. mil (español)

2.3. Los números cardinales son: $s$-ba "primero", $x$-ch-ib-al "segundo", $y$-ox-ib-al "tercero", $x$-chan-ib-al "cuarto", $x$-jo'-ob-al "quinto"; continuando así de la misma manera: + prefijo posesivo de tercera persona + Radical $+-a l$. En el caso de veintenas se agrega vink-il: $x$-chan-ib -al-vink-il "octogésimo".

\section{BIBLIOGRAFIA}

ANÓNIMO

1832 Libro en que se trata de la Lengua Tzotzil... Microfilm M. N. A.; Serie Chiapas, Rollo 70.

DE RODAZ, Fr. JUAN

1688 Arte de la Lengua Tzotzlem o Tzinacanteca con Explicación del Año Solar y un Tratado de las Quentas de los Indios en Lengua Tzotzlem.

GARCÍA DB LEÓN, ANTONIO

1970 Vocabulario y Textos de los Parajes Sisim (Chalchibuitán) y Pasté (Zinacantán). Ms. Datos de Campo.

1971 Los elementos del Tzotzil Colonial y Moderno. Cuadernos del Centro de Estudios Mayas, núm. 7; UNAM, México, 111 pp.

GuITERAS Holmes, C.

1965 Los Peligros del Alma. Visión del Mundo de un Tzotzil. Fondo de Cultura Económica, México-Buenos Aires, 307 pp.

HOPKINS, NICHOLAS A.

1967 "A Short Sketch of Chalchihuitan Tzotzil". Antbropological Linguistics, 9: 9-25.

PINEDA, EMETERIO

1845 Descripción Geográfica del Departamento de Cbiapas y Soconusco. México.

REDFIELD, ROBERT y ALFONSO VILLA ROJAS

1939 "Notes on the Ethnography of Tzeltal Comunities of Chiapas", Contributions to American Antbropology and History, vol. V, núm. 28. (Publ, of Carnegie Institution of Washington, D.C.) 


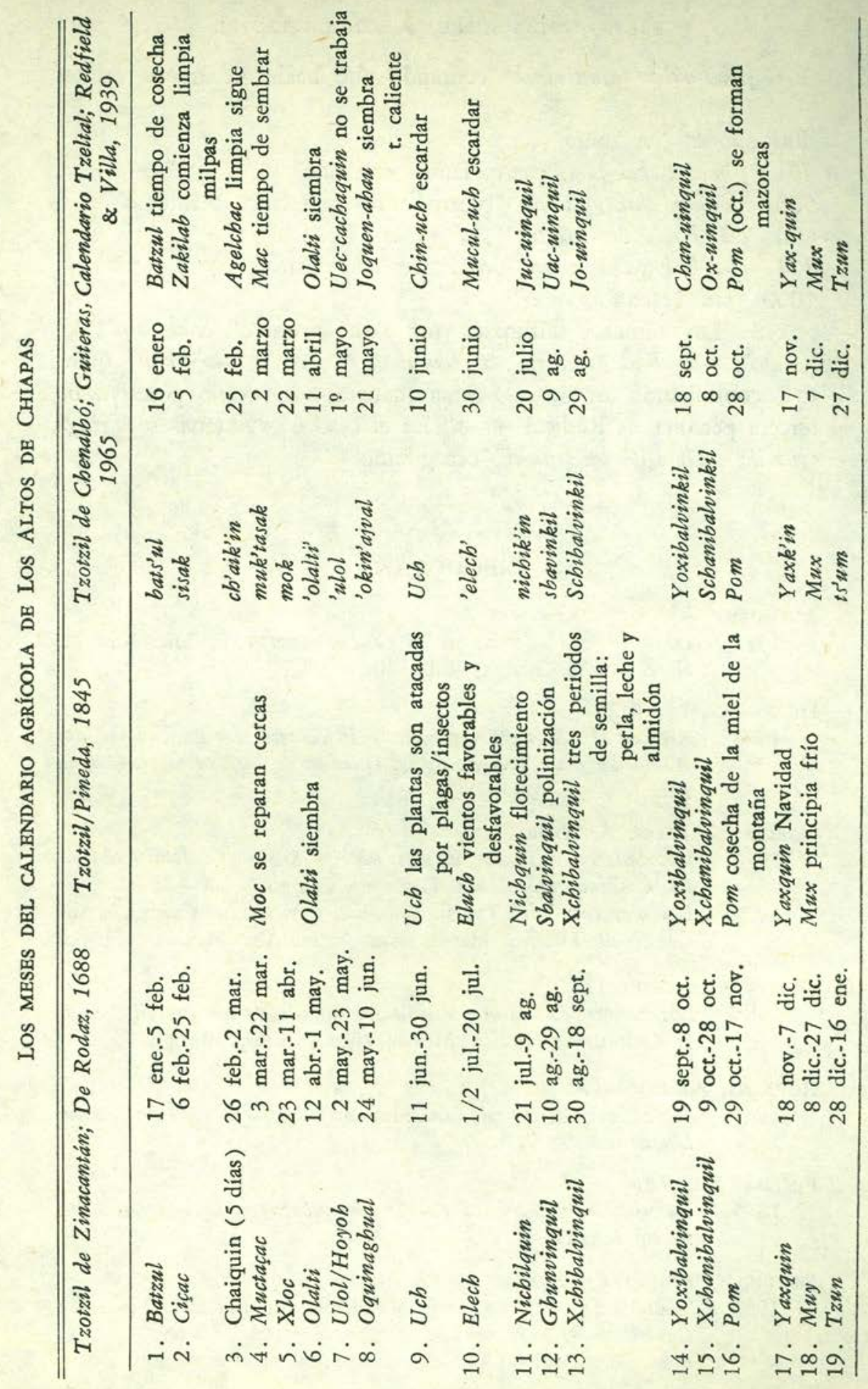

